livraisons

d'Histoire

de l'Architecture

\section{Livraisons de l'histoire de l'architecture}

$40 \mid 2020$

À propos des princes de Conti

\title{
La reconstruction des églises du Bas-Rhin à travers les archives de la société coopérative de reconstruction des églises et édifices religieux catholiques, 1945-1966
}

La reconstruction des églises du Bas-Rhin à travers les archives de la société coopérative de reconstruction des églises et édifices religieux catholiques, 1945-1966

The competition for the reconstruction of the national theater of the OpéraComique in 1893: the public architectural competition, a solution to the architectural crisis of the end of the 19th century?

Der Wiederaufbau der Kirchen im Département Bas-Rhin anhand von Quellen der Société coopérative de reconstruction des églises et édifices religieux catholiques 1945-1966

\section{Cécile Rivière}

\section{(2) OpenEdition} Journals

\section{Édition électronique}

URL : http://journals.openedition.org//ha/1326

DOI : $10.4000 /$ /ha. 1326

ISSN : 1960-5994

Éditeur

Association Livraisons d'histoire de l'architecture - LHA

\section{Édition imprimée}

Date de publication : 3 décembre 2020

Pagination : 87-98

ISSN : $1627-4970$

\section{Référence électronique}

Cécile Rivière, "La reconstruction des églises du Bas-Rhin à travers les archives de la société coopérative de reconstruction des églises et édifices religieux catholiques, 1945-1966 », Livraisons de I'histoire de l'architecture [En ligne], 40 | 2020, mis en ligne le 28 décembre 2020, consulté le 25 mars 2021. URL : http://journals.openedition.org/lha/1326 ; DOI : https://doi.org/10.4000//ha.1326 


\section{LA RECONSTRUCTION DES ÉGLISES DU BAS-RHIN À TRAVERS LES ARCHIVES DE LA SOCIÉTÉ COOPÉRATIVE DE RECONSTRUCTION DES ÉGLISES ET ÉDIFICES RELIGIEUX CATHOLIQUES, 1945-1966}

En juillet 1945, le ministère de la Reconstruction et de l'Urbanisme dénombre environ 99800 immeubles détruits ou endommagés dans le Bas-Rhin ${ }^{1}$, l'un des départements français les plus marqués par la Seconde Guerre mondiale. De nombreuses sociétés coopératives et associations syndicales de reconstruction y sont créées, conformément aux directives gouvernementales ${ }^{2}$. L'une d'entre elles ne se dédie pas à une commune ou un canton, mais aux édifices catholiques de tout le département. Elle a pour but de faciliter et de hâter leur reconstruction, notamment celle des églises; celle-ci n'est pas prioritaire, mais toutefois hautement symbolique. Dans le Bas-Rhin, les problématiques mémorielles d'un territoire fortement attaché à son identité $^{3}$ et au pittoresque de ses paysages se confrontent à celles des évolutions architecturales et liturgiques de ce second $\mathrm{XX}^{\mathrm{e}}$ siècle. L'histoire de l'Alsace en a fait un lieu d'influences culturelles françaises, allemandes et suisses, catholiques et protestantes. Au début du XX $\mathrm{XX}^{\mathrm{e}}$ siècle, le Mouvement liturgique allemand y reçoit l'accueil favorable de curés progressistes ${ }^{4}$, de même, à partir de l'entre-deuxguerres, que la revue française L'art sacré $e^{5}$ Entre traditions et modernités, les églises reconstruites sous l'égide de la Société coopérative de reconstruction des églises et édifices religieux catholiques du Bas-Rhin brossent le portrait d'une période d'innovations et de confrontations. Nous proposons de donner ici un aperçu de leur variété.

1. Archives de Paris, 52W1, carte des destructions par département, 15 juillet 1945.

2. Loi du 23 octobre 1946 sur les dommages de guerre et loi du 16 juin 1948 sur les SCR et aux ASR. Céline Frémaux, Construire des églises en France dans la seconde moitié du XXe siècle. De la commande à la réalisation. Nord-Pas-de-Calais (1945-2000), thèse d'histoire de l'art à l'Université Rennes II, sous la direction de Jean-Yves Andrieux, 2005, p. 43.

3. Jean-Marie Mayeur, "Une mémoire-frontière : l'Alsace ", in Pierre Nora (dir.), Lieux de mémoire, Paris, Gallimard, 1984, vol. I, p. 1147-1169.

4. François Wernert, Vie liturgique et mouvement liturgique en Alsace de 1900 à nos jours, Strasbourg, Ercal, 1992.

5. Françoise Caussé, La Revue "L'art sacré": le débat en France sur l'art et la religion (1945-1954), Paris, éd. du Cerf, 2010 ; Vanessa Grossman, "L'architecture religieuse dans les pages de L'art sacré", Jean-Louis Cohen, Hartmut Frank (dir.), Interférences / Interferenz, Strasbourg, Musées de Strasbourg, 2013, p. 370-379. 


\section{La Société coopérative de reconstruction des églises et édifices religieux catholiques du Bas-Rhin, acteur clé d'un territoire en chantiers}

Dans le diocèse de Strasbourg, l'accompagnement des sinistrés dans la reconstruction de leurs églises est porté par une grande figure de la charité alsacienne, le chanoine Jules Billing, un des principaux fondateurs dès mars 1945 du Groupement social des victimes de la guerre et des sinistrés d'Alsace ${ }^{6}$. Après la loi du 16 juin 1948, de ce groupement naît, dans chaque département alsacien, une Société coopérative de reconstruction des églises et édifices religieux liée à une Société coopérative de reconstitution mobilière. Dans le Bas-Rhin, le chanoine Billing devient président de la branche immobilière, épaulé par deux laïcs, le directeur Joseph Deichelbohrer et le président de la société de reconstitution mobilière, Auguste Glentzinger. Face à la complexité des opérations de reconstruction, les sociétés coopératives proposent aux communes ${ }^{7}$ et conseils de fabrique ${ }^{8}$ adhérents une prise en charge administrative et financière de leurs dossiers. Elles font aussi le lien entre les multiples acteurs impliqués dans la définition des projets, intervenant de la demande d'indemnités de dommages de guerre jusqu'à la livraison du chantier. Le dépouillement du fonds de la Société coopérative de reconstruction des églises et édifices religieux catholiques du Bas-Rhin et de son pendant mobilier, récemment versé aux Archives départementales ${ }^{9}$, permet d'éclairer un pan majeur de la reconstruction dans le département. La tâche effectuée au cours d'une vingtaine d'années par la société frappe par son ampleur: ce sont ainsi 147 églises paroissiales et 108 autres édifices catholiques qui ont été partiellement ou intégralement reconstruits.

\section{Reconstruction partielle à l'identique, "épuration " et modernité discrète des vitraux}

Plus des deux tiers des dossiers d'églises traités par la Société coopérative, soit 109 cas, sont des reconstructions partielles à l'identique. Lorsque les dégâts sont faibles ou moyens, les commanditaires ne cherchent généralement pas à faire ouvre d'invention mais à retrouver l'état antérieur de leur église, issu en majorité des XVIII et $\mathrm{XIX}^{\mathrm{e}}$ siècles. Clochers abattus, toitures ou façades percées sont reconstruits dans des techniques et matériaux traditionnels ; le mobilier liturgique d'origine est restauré, dans des dispositions pré-conciliaires. Un second autel pour la messe face au peuple y est ajouté après les réformes liturgiques du Concile Vatican II (1962-1965).

6. Catherine Maurer, "Reconstruction et construction d'églises à Strasbourg et en Alsace au milieu $\mathrm{du} \mathrm{XX}^{\mathrm{e}}$ siècle : un espace pour l'expertise urbaine dans le monde catholique? " Olivier Chatelan (dir.), Chrétiens et sociétés, $\mathrm{n}^{\circ} 21$, L'expertise urbaine dans le monde catholique francophone au XXe siècle, 2014, p. 51-63. Paul Winninger, Art sacré et nouvelles églises en Alsace: de 1945 à la fin du siècle, Strasbourg, Ercal, 1994, p. 32.

7. Généralement propriétaires de l'immobilier.

8. Généralement propriétaires du mobilier.

9. Archives départementales du Bas-Rhin (ADBR), série 2166W, 306 dossiers, versement 2016. 
Cependant, l'intérieur des églises est "épuré " dès leur reconstruction initiale, selon les idées prônées par le chanoine Vital Bourgeois dans le Bulletin ecclésiastique ${ }^{10}$, où il dénonce l'amoncellement néfaste d'objets sulpiciens hérité du XIX $\mathrm{X}^{\mathrm{e}}$ siècle. Il y recommande de recouvrir fresques et décors par une teinte neutre et unie, rejetant également la création d'œuvres picturales nouvelles, susceptibles de distraire les fidèles de l'action liturgique. Dans ces églises, l'intervention d'après-guerre est souvent repérable aux vitraux, où l'influence des mouvements précurseurs tels les Ateliers d'Art Sacré ou l'Arche, s'est imprimée plus rapidement que sur l'architecture ou l'aménagement. Les grisailles du XIX ${ }^{\mathrm{e}}$ siècle laissent place à des verrières aux couleurs vives, de maîtres-verriers alsaciens dont Ehret sur cartons du peintre Kuder, ou d'envergure nationale dont Archepel, Potet et Gaudin, les Bony. Dans les églises classées aux Monuments historiques, la beauté des vitraux modernes signés de grands noms, dont Le Chevallier à Saint-Georges de Haguenau et Ingrand à Altenstadt, répond à celle des espaces restaurés. La Société coopérative intervient peu dans ces églises, gérées par le service dédié dirigé par l'architecte en chef Bertrand Monnet et le chef d'agence des Bâtiments de France, Fernand Guri ${ }^{11}$. La Société coopérative de reconstitution mobilière est parfois sollicitée par les paroisses pour les parties non classées : mobilier, orgues.

\section{Entre tradition et modernité, une " architecture de compromis "}

Lorsque les destructions sont plus importantes voire totales, plusieurs facteurs amènent à des reconstructions différentes de l'état d'origine des églises. La principale cause n'en est pas une volonté initiale de faire œuvre modernisée, mais l'insuffisance des indemnités de dommages de guerre. Lors de faibles destructions ces indemnités sont aussi, en général, inférieures au coût réel des travaux mais l'apport demandé aux municipalités et paroisses reste raisonnable ; proportionnellement, lors de dégâts élevés, reconstituer les volumes et les détails dans leurs matériaux d'origine devient trop lourd à porter pour les villages éprouvés par la guerre. Il leur faut aussi parfois compter avec les plans de remembrement qui modifient trames viaires et alignements. Par ailleurs, ces projets où tout ou presque est à recréer sont l'occasion pour les instances de contrôle de faire valoir leurs convictions en matière d'architecture moderne. Tout au long de leur élaboration, les propositions architecturales et artistiques sont soumises à la Commission diocésaine d'art sacré, dont les secrétaires successifs, les chanoines Vital Bourgeois et Jean Ringue, portent les idées nouvelles en la matière. Les projets sont aussi présentés à l'inspecteur départemental des édifices cultuels, Fernand Guri ; à l'architecte-conseil de la construction, Charles-

10. Vital Bourgeois, "Reconstruction et aménagement des églises ", Bulletin ecclésiastique du diocèse de Strasbourg, $\mathrm{n}^{\circ}$ 66, mars 1947 , p. 76-82.

11. Nicolas Lefort, Patrimoine régional, administration nationale: la conservation des monuments historiques en Alsace de 1914 à 1964, thèse en histoire à l'Université de Strasbourg, sous la direction de François Igersheim, 2013. 
Gustave Stoskopf; et à l'architecte-conseil pour les édifices cultuels du ministère de la Reconstruction, Paul Koch. Tous trois ont engagé une réflexion sur l'église moderne et sont eux-mêmes auteurs de projets innovants. Les avis de ces quatre instances, en faveur de la sobriété, prônant l'usage des nouvelles techniques, vont dans le sens des économies nécessitées par les faibles budgets. Ils se heurtent cependant souvent à l'incompréhension voire au refus catégorique des maires et curés qui cherchent à redonner aux habitants un cadre de vie proche de celui perdu par faits de guerre. La Société coopérative joue alors le rôle de conciliateur, organisant visites et réunions, travaillant de concert avec les architectes-reconstructeurs à aboutir à un compromis ${ }^{12}$. L'architecture résultant de ce processus présente des traits traditionnels, à même d'évoquer l'image archétypique de l'église de village, mais dans une expression et des techniques constructives modernes. Ces reconstructions débutent plus tardivement, en 1951 pour les premières d'entre elles et à la veille du Concile Vatican II pour la majorité. L'aménagement intérieur témoigne aussi d'une conception d' " entre-deux ». Il se conforme au Directoire d'art sacré diffusé par l'évêché en 1955, où, parmi les modernisations annonçant les réformes à venir, l'autel est prévu « de telle manière qu'il permette la célébration aussi bien face à l'abside que face au peuple " ${ }^{13}$. Il demande aussi la mise en valeur des cuves baptismales au sein d'espaces dédiés, à l'entrée de l'église ou dans la base du clocher, faisant suite aux réformes de Pie XII. Les autels latéraux et le banc de communion, malgré un dessin sobre, restent cependant présents dans une disposition traditionnelle. Certaines de ces églises sont réaménagées peu de temps après, suite à la promulgation en 1963 de la Constitution sur la Sainte Liturgie.

\section{Les églises blanches du Nord de l'Alsace}

Parmi ces cas de destruction grave, dix sont regroupés dans une zone rurale durement marquée par les combats tardifs de libération, au Nord de l'Alsace. Les églises néo-classiques et néo-médiévales sinistrées y sont reconstruites dans une grande homogénéité d'ensemble, éloignée de leurs styles d'origine. Toutes ont en commun la blancheur de leurs façades et leur géométrie épurée. Certains éléments tels les baies affirment une modernité dépouillée, d'autres se raccrochent à la tradition - plan à nef rectangulaire et chœur en saillie, toiture à deux pans, entrée encadrée de grès des Vosges ou abritée par un auvent à tuiles plates. Si la gravité des sinistres et l'insuffisance des indemnités expliquent la nécessaire sobriété de l'ensemble, son homogénéité est quant à elle liée à la prédominance des œuvres de deux architectes, Roger Pillon et Henry Walker. Les premières reconstructions, entre 1951 et 1960, présentent la silhouette rassurante de l'église de village avec clocher axial à flèche en pavillon. Il en va ainsi à Cleebourg-Bremmelbach (Pillon

12. L'“ apparente liberté " doublement "surveillée » des architectes-reconstructeurs est également soulignée par Alain Nafilyan, "La reconstruction des édifices religieux en Basse-Normandie après la Seconde Guerre mondiale ", In Situ, revue des patrimoines [en ligne: https://journals.openedition .org/insitu/4893] nº 11, 2009, p. 44.

13. Commission diocésaine d'art sacré, "Directoire d'art sacré ", supplément au Bulletin ecclésiastique du diocèse de Strasbourg, $\mathrm{n}^{\circ} 75,1955$. 


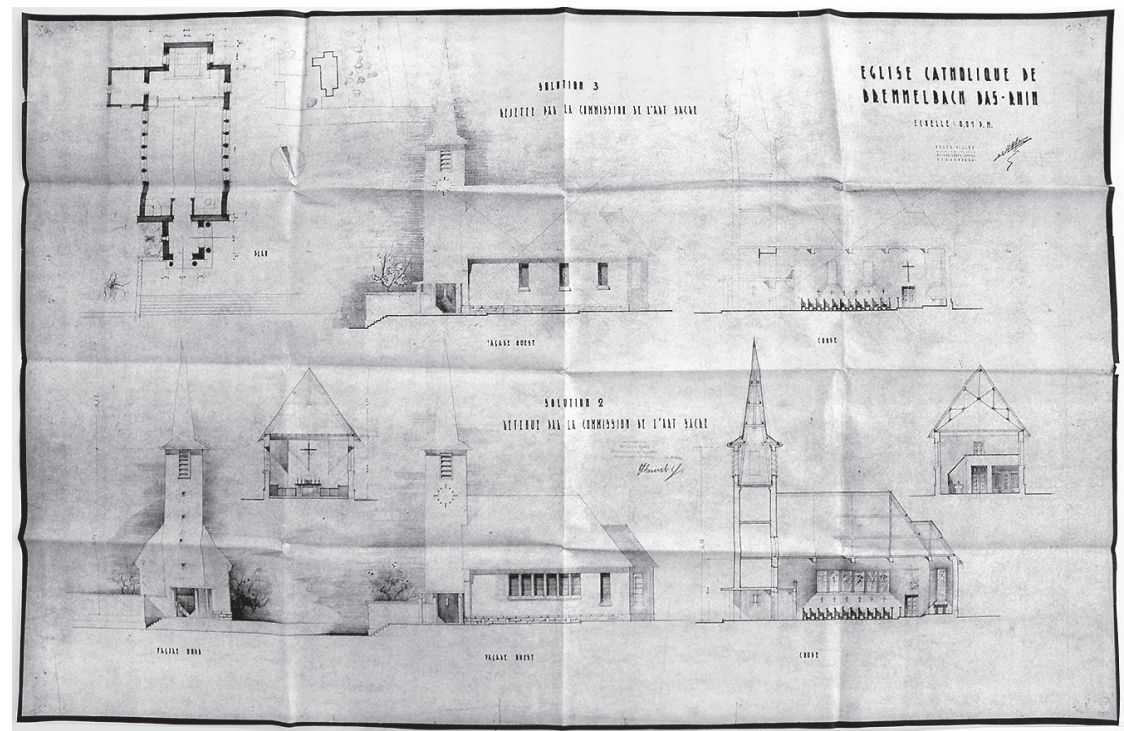

Ill. 1 : ADBR, 2166W23, "Église catholique de Bremmelbach Bas-Rhin », plans, en partie haute façade rejetée par la Commission diocésaine d'art sacré, Roger Pillon arch., n.d. [1953]. (C) Cl. Cécile Rivière.

arch., 1953-1956) où le curé Maurer déplore toutefois de ne pas être entendu par l'architecte et la Commission diocésaine d'art sacré : «Mr Pillon n’est pas content de ce que nous tenons (tout Bremmelbach derrière moi) à ce que l'église soit reconstruite dans sa forme originale " ${ }^{14}$, "la Commission a approuvé les plans présentés par Mr Pillon. (...) L'avis contraire de M. le Maire, de moi-même ne compte pas - on passe outre ${ }^{15}$ (ill. 1).

Le temps passant, les flèches se font plus fines, se transformant en pointes de cuivre effilées (Stundwiller, Walker arch., 1962-1965) voire disparaissant (Scheibenhard, Delacourt arch., 1962-1965). Le changement majeur de cette seconde période, entre 1960 et 1965, réside en la séparation de la nef et du clocher, devenant campanile isolé. Ce "véritable leitmotiv de l'architecture religieuse moderne " ${ }^{16}$ trouve parfois une expression issue d'un modèle historique régional, le clocher en bâtière (Buhl, Walker arch., 1960-1965 ; Oberrœedern, Walker arch., 1962-1963 ; Dambach-Neunhoffen, Carrière arch., 1964). À Buhl, l'architecture de la nef reconstruite fait le lien avec le chœur néo-médiéval par sa volumétrie et le marquage des travées, reprises de l'état antérieur. Le curé Helleisen et le maire y rejettent les décisions de Paul Koch par des mots lapidaires : "Nous n’aimons pas le Moderne ${ }^{17}$ (ill. 2).

14. ADBR, 2166W23, courrier du 28 septembre 1951 du curé Maurer à J. Deichelbohrer.

15. ADBR, 2166W23, courrier du 23 février 1953 du curé Maurer à J. Deichelbohrer.

16. Dave Lüthi, "Un paysage sans clocher est un paysage décapité". Tours et beffrois en Suisse romande, 1848-1939", Art+Architecture en Suisse, vol. 58, n 2, 2007, p. 48.

17. ADBR, 2166W26, courrier du 15 octobre 1957 du curé Helleisen au chanoine Billing. 


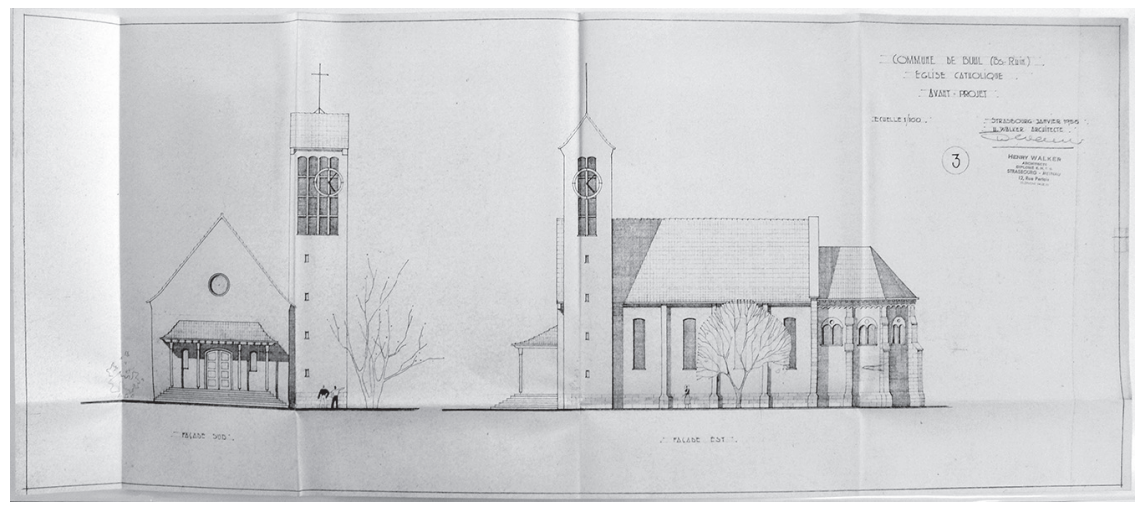

Ill. 2 : ADBR, 2166W25, "Commune de Buhl, Bas-Rhin, église catholique - avant-projet ", élévations Sud et Est, Henry Walker arch., janvier 1958. (C) Cl. Cécile Rivière.

L'influence protestante est manifeste : dans les mêmes années, dix églises luthériennes et temples réformés sont reconstruits dans ce secteur avec une architecture tout à fait similaire, parfois par Roger Pillon. Les lieux de culte protestants anciens, présents par dizaines dans cette région relèvent, historiquement, d'un caractère plus sobre que les églises voisines catholiques : le modèle est d'un néo-roman dépouillé, aux façades blanches soulignées par de simples chaînages d'angles. Cette simplicité de conception, qui a prévalu dans la reconstruction des édifices protestants, a pu se révéler, de même, pertinente face aux problématiques de la reconstruction catholique. Loin des manifestes de la modernité franche, cet ensemble rejoint ainsi les réalisations bâties dès l'entre-deux-guerres dans d'autres régions peu urbanisées et pluriconfessionnelles, en Allemagne ${ }^{18}$ et en Suisse romande ${ }^{19}$.

\section{Grès des Vosges et béton armé}

En dehors de ce secteur d' "églises blanches ", les réalisations sont plus variées. Quatre d'entre elles proposent différentes mises en œuvre du matériau emblématique de la région, le grès des Vosges, entre tradition et modernité. Elles permettent aussi d'interroger le rapport créé entre église et espace public. À Bitschhoffen (Schwab arch., 1952-1957), l'église intégralement détruite est déplacée sur le point culminant du village et est réalisée en moellons de récupération apparents. Dans le village voisin, Kindwiller, la reconstruction de l'église (Guri arch., 1960-1965) est un exercice de longue haleine pour l'architecte. De 1952 à 1957, ses propositions variées cherchent à trouver un point d'entente entre les services de l'urbanisme demandant un recul sur alignement, la Commission diocésaine d'art sacré et les architectesconseil favorables à un clocher isolé latéral et les commanditaires locaux refusant

18. Par exemple : église de Scheibenhardt, à deux pas de l'Alsace, années 1930 ; œuvres de Josef Fehlig jusqu'aux années 1950 .

19. Par exemple : œuvres de Fernand Dumas ; église de Renens (Lavenex arch., 1932) à la "modernité très tempérée" (Dave Lüthi, op. cit., p. 47). 
de reconstruire autrement que sur les fondations existantes ${ }^{20}$. Après une "réunion extrêmement mouvementée " ${ }^{21}$ organisée par la Société coopérative en 1957, le maire et le curé obtiennent gain de cause. Le clocher est reconstruit dans l'axe de la nef; une maison voisine est supprimée pour créer le parvis. Le résultat final, " expression du caractère de ces gens de terroir » ${ }^{22}$ selon Guri qui s'est résolu à contrecœur à cette solution, mêle structure apparente de béton armé et revêtement de grès rose. Le cas de Friesenheim (Herrenschmidt arch., 1951-1955) est initialement similaire, mais l'issue en est différente. Le plan d'urbanisme demandant une zone de reculement, une travée de la nef ancienne est supprimée et le clocher déplacé latéralement. À Marckolsheim (Horn et Sigrist arch., 1961-1965), c'est l'église entière qui est déplacée pour se conformer au plan d'urbanisme, avec le même principe de clocher latéral sur un espace public dégagé. En dehors de Kindwiller et contrairement aux églises plus dépouillées évoquées précédemment, ces projets montrent une coopération en bonne entente entre commanditaires locaux et instances de contrôle, autour des plans proposés par les architectes-reconstructeurs. Ces derniers ont su y trouver une volumétrie et une matérialité en lien avec les traditions locales, dans une expression sobre et sensible.

\section{Modernités}

La «table-rase » des villages hautement sinistrés ne mène que rarement au choix de la franche modernité : seules huit églises s'éloignant résolument des typologies traditionnelles et régionales ont été reconstruites sous l'égide de la Société coopérative, dont deux en Alsace du Nord et six le long du Rhin. Elles mêlent les influences d'églises modernes de référence, issues de voyages et des revues spécialisées. Celles-ci, outre des photographies, publient les plans de nouvelles dispositions architecturales et liturgiques. Malgré la diffusion d'exemples variés d'églises à plans centrés dès l'entre-deux-guerres ${ }^{23}$, les églises modernes reconstruites dans le Bas-Rhin se réferent au modèle de l'église-halle ou Wegkirche. Ce type, à grande nef unique en longueur, chœur de même largeur et campanile isolé, "tend à devenir une mode " ${ }^{24}$ dans la reconstruction pré-conciliaire, à travers toute l'Europe ${ }^{25}$. Sa forte prédominance dans le Bas-Rhin n’y empêche ni la diversité des expressions architecturales, ni l'évolution des recherches liturgiques.

20. Officiellement pour raisons de coût ; mais cet avis est maintenu lorsque le ministère annonce prendre en charge les fondations d'un nouveau clocher, montrant la volonté tacite des commanditaires locaux de reconstituer l'ancienne silhouette de l'église.

21. ADBR, 2166W102, courrier du 9 juin 1958 du chanoine Billing à Mgr l'Évêque Weber.

22. ADBR, 2166W102, courrier du 18 janvier 1957 de Fernand Guri à Paul Koch.

23. Lucile Pierron, "À la recherche d'un plan" : nouvelles compositions architecturales à l'heure de Vatican II ", FabricA, n ${ }^{\circ} 7,2013$, p. 10-35.

24. L'Art sacré, no 3-4 "Les bases d'une étude critique ", novembre-décembre 1956, p. 6.

25. Franck Debié et Pierre Vérot, Urbanisme et art sacré, Paris, Criterion, 1991, p. 254. 


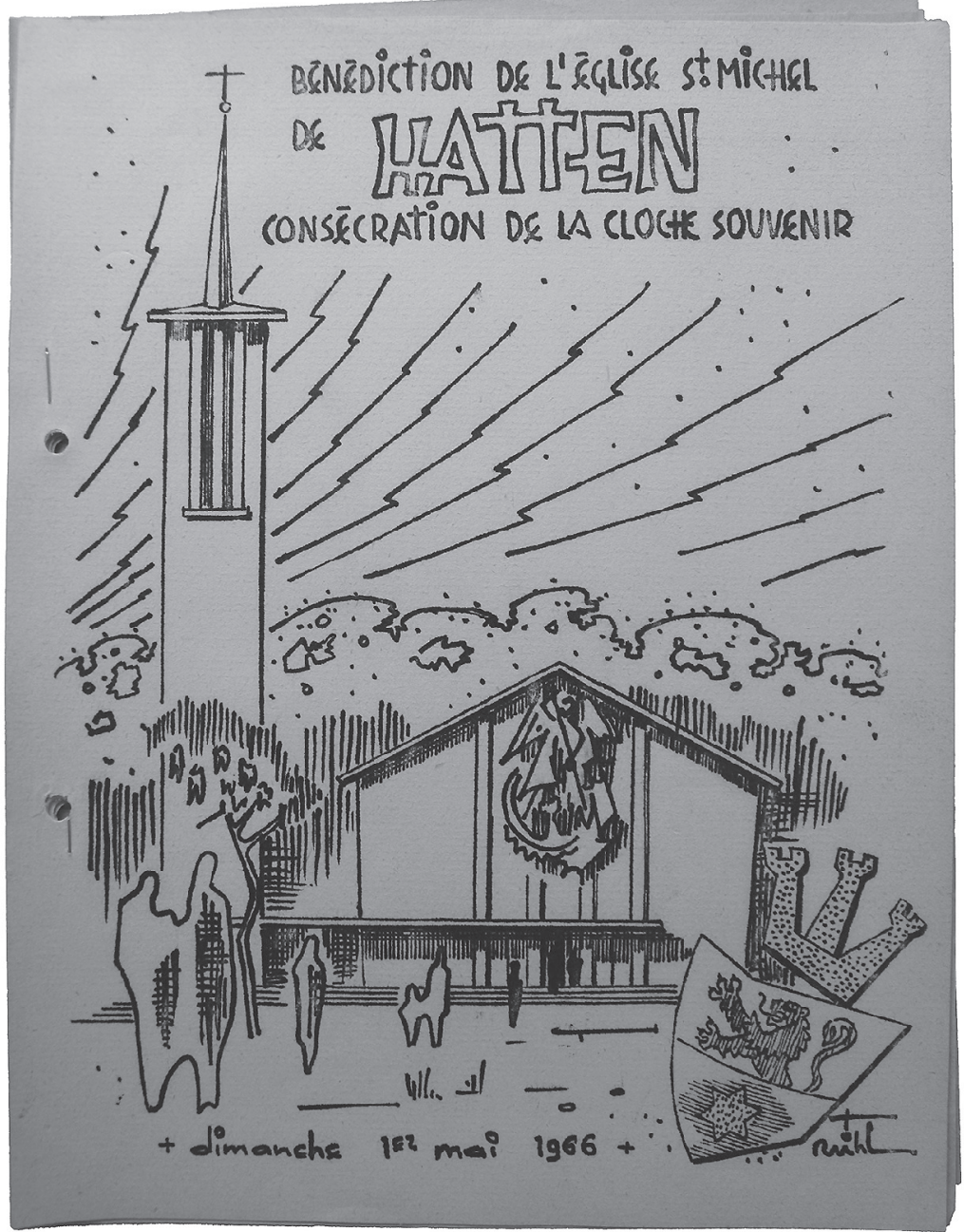

Ill. 3 : ADBR, 2166W81, Bénédiction de l'église St-Michel-de-Hatten, consécration de la cloche souvenir, dimanche $1^{\text {er }}$ mai 1966, couverture du livret, Tristan Ruhlmann, Roger Pillon arch., 1966. (C) Cl. Cécile Rivière.

\section{Variations sur le modèle de l'église-halle}

Les deux exemples d'Alsace du Nord, à Hatten (1962-1966) et Rittershoffen (1961-1963) sont les dernières réalisations alsaciennes de Roger Pillon. Leur reconstruction tardive est liée à la complexité des opérations : dans chaque village, les indemnités de dommages de guerre sont divisées en deux pour reconstruire une église catholique et une église protestante là où, avant-guerre, n'existait qu'une seule église simultanée. Pillon y abandonne les typologies traditionnelles mises en œuvre jusqu'alors. Les deux édifices s'inscrivent toutefois pleinement dans son œuvre : 
façades lisses de teinte claire, campanile massif à fine flèche de cuivre. Ces églises sont intéressantes pour le lien visuel et typologique qu'elles proposent, au cour des villages, avec l'église protestante qui leur fait face. Les espaces intérieurs, grands et vides, sont animés par les vitraux colorés de Tristan Ruhlmann. À Rittershoffen, la verrière du pignon se place sous la bannière de l'œcuménisme. À Hatten, la Commission diocésaine d'art sacré considère que l'église est d'une "simplicité trop profane et dépourvue de grandeur ${ }^{26}$; Stoskopf appelle à une " composition [sculptée] largement développée » ${ }^{27}$ sur le pignon. Un groupe de Saint-Michel est étudié par Raymond Keller et esquissé sur le livret de bénédiction, mais ne sera jamais réalisé faute de moyens financiers (ill. 3).

L'animation du pignon par une œuvre sculptée, rompant l'austérité générale des projets, est une constante dans les trois autres exemples d'églises-halles. À Gambsheim (Guri arch., 1953-1959) Stentzel et Stoll représentent les quatre évangélistes en grès rose ; la première église véritablement moderne consacrée dans le Bas-Rhin est louée par Vital Bourgeois pour sa "noblesse", son " ordonnance simple et authentique ", sa "logique rigoureuse, au service de la vérité » ${ }^{28}$. À Seltz (Viallefond arch., 1953-1962), les premiers projets prévoient un arc parabolique monumental en façade. Elle est finalement entièrement occupée par la Transfiguration sculptée sur ciment frais par Lambert-Rucki. Y retrouver l'auteur du chemin-de-croix de la basilique de Blois n'est pas tout à fait un hasard : celle-ci, très publiée dans les revues d'architecture et d'art sacré, est prise comme référence par Viallefond qui en reprend les volumétries et le rythme des façades latérales. Il fait lui-même appel à LambertRucki lors du concours pour la sculpture. La référence du clocher, réalisé plus tardivement, est à chercher du côté de la Suisse, où Viallefond voyage juste avant de le concevoir ${ }^{29}$. Les esquisses reprenant fidèlement celui de l'église Bruder-Klaus d'Oberwil sont légèrement modifiées sur avis du Père Jean Capellades, consulté par l'architecte. L'intérêt de ce projet, œuvre savante de collage d'influences variées, se trouve aussi dans la conservation du chœur et des chapelles gothiques, auxquels se marient judicieusement les éléments modernes (ill. 4).

La conservation du chœur gothique est aussi au centre du projet de reconstruction de l'église de Rhinau (Chirot, Kuntz, Schaetzel arch., 1955-1961). Sa conception est plus longue et prend quelques détours étonnants. À la demande du curé Deyber, les premières esquisses proposent une vaste nef ovale, "pour raisons liturgiques ${ }^{30}$. L'ancien chœur y est accolé en tant que chapelle. Stoskopf et Guri déplorent le manque de logique et le coût élevé du projet ; le chanoine Bourgeois recommande une nef rectangulaire. Un voyage en Suisse est organisé en 1954 avec les commanditaires ; le projet reprend ensuite les lignes de l'église de Tous-les-Saints de Bâle (Baur arch., 1947-1951). S’y retrouvent la longue nef à files de colonnes, le bas-côté abritant le baptistère, une chapelle de semaine et la tribune des chantres,

26. ADBR, 2166W81, courrier du 21 janvier 1956 de J. Deichelbohrer au maire.

27. ADBR, 2166W81, courrier du 5 octobre 1956 de R. Pillon au curé Riegert.

28. ADBR, 2166W60, livret de consécration de l'église de Gambsheim, p. 11-15.

29. ADBR, 2166W208, courrier du 16 avril 1955 de J. Viallefond à J. Deichelbohrer.

30. ADBR, 2166W173, compte rendu de réunion du 17 mars 1954. 


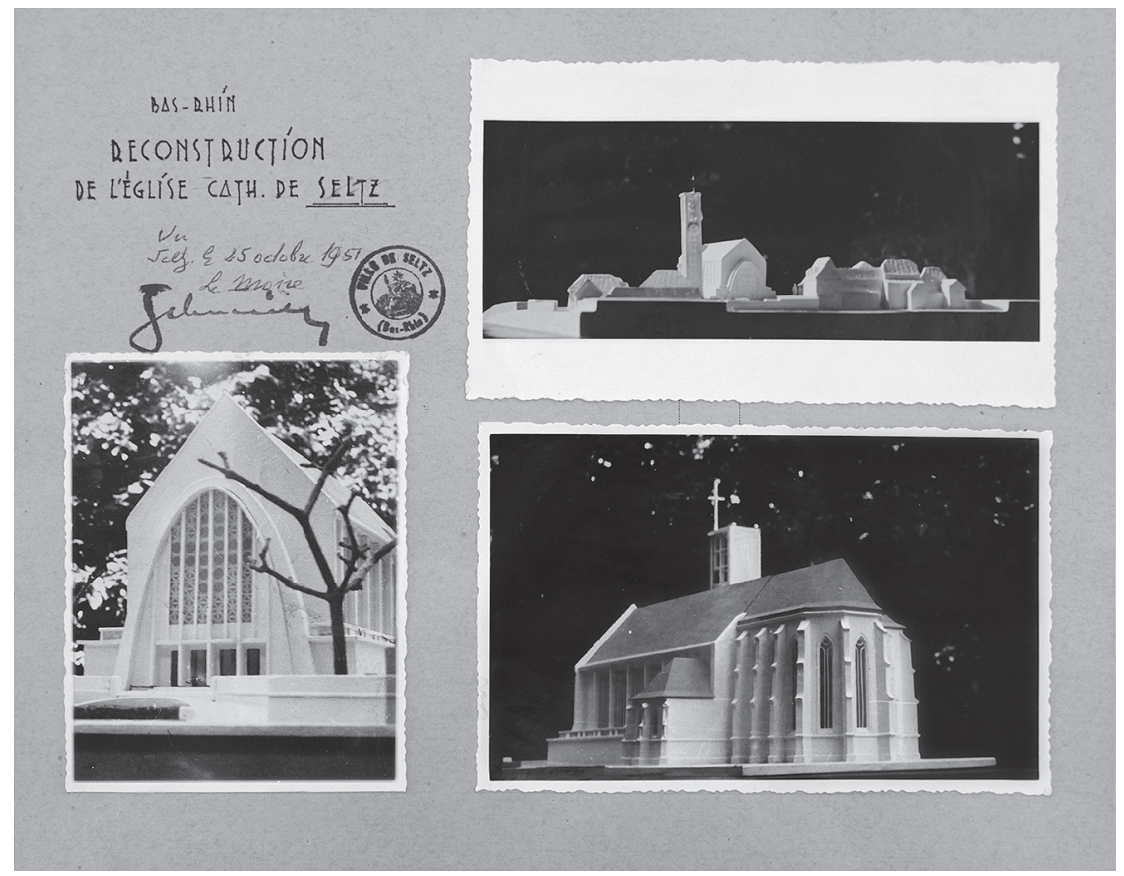

Ill. 4: ADBR, 2166W211, "Bas-Rhin - reconstruction de l'église cath. de Seltz ", planche photographique des maquettes, projet modifié ultérieurement, Jean Viallefond arch., octobre 1951. (C) Cl. Cécile Rivière.

ainsi que le haut campanile ajouré et le motif sculpté surplombant l'entrée principale (Henninger sculpt.). L'aspect local y est apporté par le parement en grès des Vosges en façades et au mur du chevet. L'ancien chœur est traité en objet isolé, à valeur mémorielle, abritant le monument aux morts (ill. 5).

\section{Innovations liturgiques et typologiques}

L'aménagement intérieur des églises-halles bas-rhinoises montre une attention aux évolutions liturgiques de la période : autel au centre du chœur, ambon, absence de banc de communion et d'autels latéraux. Les plans de l'église de Rhinau, précédant l'encyclique de Pie XII Musicae sacrae, innovent en plaçant au droit du chœur la tribune des chantres et l'orgue. Cette disposition, ainsi que la chapelle de semaine dans le bas-côté unique, annoncent les particularités de l'« église du Concile " ${ }^{31}$ à Offendorf (Schulé arch., 1960-1962). Tout en rebâtissant sur les anciennes fondations et la base du clocher conservée, le curé liturgiste Henri Oster amène cette église au rang de modèle d'aménagement moderne. Les trois pôles de la célébration, autel, ambon et siège du célébrant sont réalisés en marbre dans un chœur dégagé.

31. Paroles de Mgr Elchinger lors de la consécration, cité par Adrien Schneider, "Un joyau du Concile ", Almanach Sainte-Odile, 2014, p. 133. 


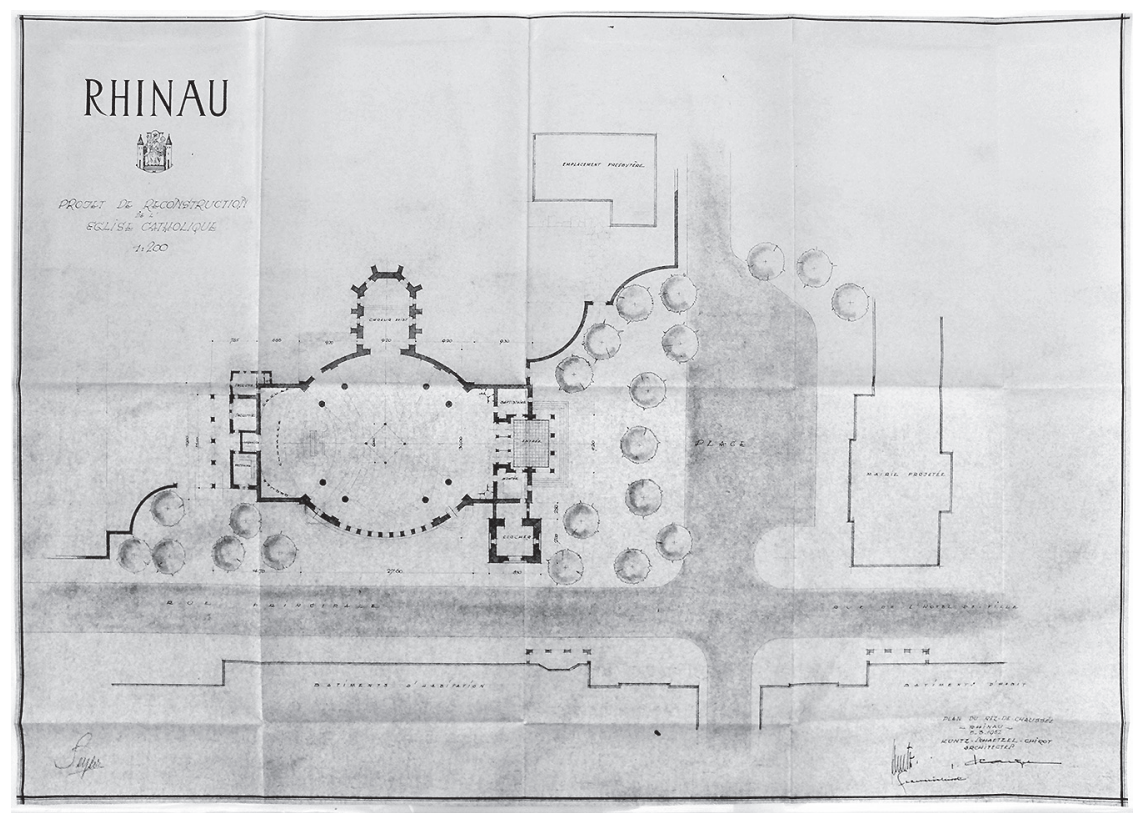

Ill. 5 : ADBR, 2166W172, " Rhinau - projet de reconstruction de l'église catholique ", plan du rez-dechaussée non réalisé, Ernest Kuntz, Simon Schaetzel et Francis Chirot, arch., mars 1952. C Cl. Cécile Rivière.

Le tabernacle est placé sur l'autel de la chapelle latérale, d'où il reste visible pour l'assemblée de la nef principale. Ces dispositions annonciatrices des réformes à venir valent à l'église de figurer dans la très officielle revue vaticane Notitiae ${ }^{32}$, parmi de rares exemples pour l'application de la Constitution sur la Sainte Liturgie. Les deux réalisations les plus tardives, construites pendant et après le Concile, à Fort-Louis (Guri arch., 1963-1971) et Herrlisheim (Guri et Monnet arch., 1965-1971) résultent d'un long processus de projet. Les églises, classées monuments historiques avant-guerre, sont jugées in fine non réparables et déclassées. Les deux architectes des monuments historiques gardent la main pour l'élaboration de projets modernes. À Fort-Louis, Guri, en architecte érudit, mêle évocation de la cité historique conçue par Vauban et franche modernité dans un volume massif de grès rose. À Herrlisheim ${ }^{33}$, les architectes conçoivent le parti le plus novateur de la reconstruction dans le Bas-Rhin, où les géométries imbriquées créent des espaces flexibles et fluides. Le positionnement du baptistère au droit du chœur y représente une

32. Commission de mise en œuvre de la Constitution sur la sainte liturgie, Notitiae, [en ligne : http:// www.cultodivino.va/content/cultodivino/it/rivista-notitiae/indici-annate/1967/34.html] $\mathrm{n}^{\circ} 34$, octobre 1967 , p. 325.

33. Jokine Wehbé, «Une reconstruction controversée : l'église Saint-Arbogast de Herrlisheim (19451972)"Revue d'Alsace [en ligne : http://journals.openedition.org/alsace/2416] n 142, 2016, p. 207228. 
dernière évolution vers une liturgie actualisée. La Société coopérative ne suit qu'une part mineure de ces reconstructions. Cela est dû à la présence des architectes des monuments historiques, bien au fait du déroulé des opérations et à leur réalisation tardive, outrepassant la dissolution de la société qui a lieu en 1966.

Avant la création par l'évêché de Strasbourg, en 1963, de la Commission diocésaine de construction chargée des paroisses nouvelles, la Société coopérative de reconstruction a à suivre quelques projets de construction neuve. À Diemeringen, les paroissiens doivent se rendre dans le village voisin jusqu'à la construction de leur église (Lavandier arch., 1959-1961). Elle est saluée pour «son idée créatrice, sa simplicité et sa franchise " avec un "maximum d'intensité " ${ }^{4}$, correspondant à la modestie prônée par Jean Capellades ${ }^{35}$. Les églises centrées trouvent leur place dans les périphéries strasbourgeoises, au Sacré-Cœur de la Montagne-Verte elliptique (De Lapparent arch., 1958-1960) et à l'église circulaire Saint-Bernard (Monserat et Schwab arch., 1960-1962). À la Meinau, Saint-Vincent-de-Paul (Le Donné arch., 1962-1964) reprend le modèle de l'église-halle. La rigueur de l'œuvre de l'élève de Perret y contraste avec les vitraux aériens et organiques de Léon Zack. Par la suite, la Commission diocésaine de construction est présidée par Jules Billing, qui continue ainsi son œuvre de bâtisseur. Aux efforts de la reconstruction succèdent ceux de l'équipement des banlieues. Vingt-trois églises catholiques nouvelles sont réalisées dans le Bas-Rhin, dont la grande diversité illustre les courants successifs de pensée et d'architecture catholiques. La dernière d'entre elles, à l'Elsau, s'achève en 1993.

Cécile RIVIÈRE

Doctorante en histoire de l'architecture, UR3400 Arche, Université de Strasbourg

34. ADBR, 2166W37, texte non daté [discours de bénédiction, 13 novembre 1960].

35. Jean Capellades, Guide des églises nouvelles en France, Paris, éd. du Cerf, 1969. 